\title{
cAMP-Dependent Axon Guidance Is Distinctly Regulated by Epac and Protein Kinase A
}

\author{
Andrew J. Murray, Steven J. Tucker, and Derryck A. Shewan \\ Institute of Medical Sciences, School of Medical Sciences, College of Life Sciences and Medicine, University of Aberdeen, Forresterhill, Aberdeen AB25 2ZD, \\ United Kingdom
}

cAMP is a key mediator of a number of molecules that induce growth cone chemotaxis, including netrin-1 and myelin-associated glycoprotein (MAG). Endogenous neuronal cAMP levels decline during development, and concomitantly axonal growth cones switch their response to cAMP-dependent guidance cues from attraction to repulsion. The mechanisms by which cAMP regulates these polarized growth cone responses are unknown. We report that embryonic growth cone attraction to gradients of cAMP, netrin-1, or MAG is mediated by Epac. Conversely, the repulsion conferred by MAG or netrin-1 on adult growth cones is mediated by protein kinase A (PKA). Furthermore, fluorescence resonance energy transfer reveals that netrin-1 distinctly activates Epac in embryonic growth cones but PKA in postnatal neurons. Our results suggest that cAMP mediates growth cone attraction or repulsion by distinctly activating Epac or PKA, respectively. Moreover, we propose that the developmental switch in growth cone response to gradients of cAMP-dependent guidance cues from attraction to repulsion is the result of a switch from Epac- to PKA-mediated signaling pathways.

\section{Introduction}

During nervous system development, neuronal growth cones detect environmental cues that cooperate to guide axons to their appropriate targets. Axon guidance cues induce attraction or repulsion depending on neuronal type, age, or the balance of signaling activity within the growth cone (TessierLavigne and Goodman, 1996; Huber et al., 2003). Netrin-1 and myelin-associated glycoprotein (MAG), for example, attract and repel axons depending on intracellular levels of cAMP. High levels mediate attraction, whereas lower levels result in repulsion (Ming et al., 1997; Song et al., 1998; Song and Poo, 1999; Shewan et al., 2002). In development, rat DRG neurons exhibit a decline in intracellular cAMP levels (Cai et al., 2001) that is believed to underlie a switch in response to netrin-1 and MAG. The axon growth promotion and growth cone attraction conferred on embryonic neurons change to axon growth inhibition and growth cone repulsion in older neurons. This is of great consequence to the injured adult mammalian CNS because axon regeneration is inhibited, resulting in loss of function.

The mechanisms that transduce cAMP signals to elicit specific growth cone responses to extracellular guidance cues are not fully known. Until recently, cAMP was thought to signal solely

Received June 25, 2009; revised 0ct. 8, 2009; accepted 0ct. 14, 2009.

This work was supported by grants from the Medical Research Council and Tenovus Scotland to D.A.S. We thank Andrew Peace for assistance in the laboratory and Prof. Graeme Nixon for guidance in $\mathrm{Ca}^{2+}$ imaging. We are grateful to Prof. Manuela Zaccolo (University of Glasgow, Glasgow, UK), Dr. Gabrielle Berger (Leica Microsystems, Mannheim, Germany), and Paul McCormack (Leica Microsystems, Milton Keynes, UK) for discussions on this manuscript.

Correspondence should be addressed to either Andrew J. Murray or Derryck A. Shewan, Institute of Medical Sciences, School of Medical Sciences, College of Life Sciences and Medicine, University of Aberdeen, Forresterhill, Aberdeen AB25 2ZD, UK, E-mail: a.j.murray@abdn.ac.uk or d.shewan@abdn.ac.uk.

DOI:10.1523/JNEUROSCI.3071-09.2009

Copyright $\odot 2009$ Society for Neuroscience $\quad$ 0270-6474/09/2915434-11\$15.00/0 through protein kinase A (PKA), which has therefore been implicated in mediating both growth cone attraction and repulsion (Ming et al., 1997; Song et al., 1997; Song and Poo, 1999; Han et al., 2007). However, it is not known how these polarized responses exerted by guidance cues such as netrin-1 and MAG can be mediated solely through PKA signaling. Epac, a guanine nucleotide exchange factor for the small G-protein Rap1, was identified recently as a novel protein that can be directly activated by cAMP, independently of PKA (de Rooij et al., 1998; Kawasaki et al., 1998). We have shown that Epac mediates cAMP-dependent axon growth and regeneration by rat DRG neurons and that asymmetric activation of Epac induces growth cone attraction similar to that seen using cAMP agonists (Murray and Shewan, 2008), but the relative contributions of Epac and PKA in axon guidance remain to be explored. In this study, we ask whether PKA and Epac collaborate in transducing cAMP-dependent axon guidance signals in rat DRG neurons or whether they have distinct roles to play. Remarkably, we find that Epac mediates growth cone attraction to gradients of cAMP, MAG, or netrin-1, but the growth cone repulsion inflicted by MAG and netrin-1 on adult neurons is mediated by PKA. Using live fluorescence resonance energy transfer (FRET) imaging, we also show that netrin-1 activates Epac but not PKA in embryonic growth cones but then switches to activating PKA and not Epac in postnatal growth cones. We also show that Epac is required for B-Raf activation and netrin-1-induced calcium elevation. We propose that the polarized responses of growth cone attraction and repulsion effected by cAMP-dependent guidance cues are mediated distinctly by Epac and PKA signaling pathways, respectively, and that the developmental switch in response to key axon guidance cues from attraction to repulsion is the result of a switch from Epac to PKA activation. 


\section{Materials and Methods}

Animals. Sprague Dawley rats were used at three progressive stages of development: embryonic days $14-16$, postnatal days $1-3$, and adult $(>3$ months).

Reagents. Sp-8-Br-cAMPS (Sp-cAMPS), 8-CPT-2'-O-Me-cAMP (8Me-cAMP), 6-Phe-cAMP, and 8-Mb-cAMP were purchased from Biolog Life Sciences Institute. MAG-Fc fusion protein and netrin-1 protein were kind gifts from Prof. Marie T. Filbin (Hunter College, New York, NY) and Prof. Timothy E. Kennedy (McGill University, Montreal, Quebec, Canada), respectively.

Calcium imaging. Embryonic or adult DRG neurons plated on $2 \mu \mathrm{g} / \mathrm{ml}$ laminin were loaded with $4 \mu \mathrm{M}$ fura-2 AM for $60 \mathrm{~min}$ in modified Bottenstein and Sato's fluid (BSF) at $37^{\circ} \mathrm{C}$, followed by a $30 \mathrm{~min}$ incubation in BSF. Growth cones were imaged on a Zeiss Axiovert 200 inverted microscope equipped with a cooled CCD camera and a polychromatic illumination system. Fluorescence images were captured at 340 and 380 $\mathrm{nm}$, and the ratio of fluorescence intensity was calculated after background subtraction using Metafluor 4.6 software. Results are expressed as percentage change in the $340 / 380$ ratio after drug application.

DRG neuron culture. Rat DRGs were collected in Ham's F12 culture medium (Invitrogen) and trimmed of roots. Embryonic and neonatal ganglia were transferred to a $1 \mathrm{ml}$ papain solution $(10 \mathrm{U} / \mathrm{ml}$; Lorne Laboratories) in $15 \mathrm{~mm}$ HEPES (Sigma) buffered HBSS (Invitrogen) containing $0.2 \mathrm{mg} / \mathrm{ml}$ cysteine (Sigma) and incubated at $37^{\circ} \mathrm{C}$ for $20 \mathrm{~min}$. The tissue was then transferred to $100 \mu \mathrm{l}$ of HBSS containing $0.25 \mathrm{mg} / \mathrm{ml}$ trypsin inhibitor (Sigma, UK) and $50 \mu \mathrm{g} / \mathrm{ml}$ DNase (Sigma, UK; TID) and triturated using a Gilson P200 pipette (Anachem) until a single-cell suspension was achieved. The dissociated neurons were diluted to a concentration of $4000 / \mathrm{ml}$ in BSF supplemented with nerve growth factor (100 ng/ml; Serotec) and insulin $(20 \mu \mathrm{g} / \mathrm{ml}$; Sigma). Neurons that were to be transfected with small interfering RNA (siRNA) were pelleted at $2000 \mathrm{rpm}$ for $5 \mathrm{~min}$ in a microcentrifuge and resuspended in Amaxa Nucleofector solution (Lonza). Adult ganglia were incubated for $1 \mathrm{~h}$ at $37^{\circ} \mathrm{C}$ in $0.125 \%$ collagenase (Sigma) before transfer to a papain solution as described above. The ganglia were triturated in BSF before being spun in $1 \mathrm{ml}$ volumes at $100 \mathrm{~g}_{\max }$ through a cushion of $2 \mathrm{ml}$ of $15 \%$ BSA to remove debris. The cell pellet was resuspended in TID and diluted in BSF to a concentration of 2000 cells/ml. For siRNA and cDNA transfections, neurons were resuspended in $100 \mu \mathrm{l}$ of Nucleofector solution and transfected using an Amaxa Nucleofector device (Lonza).

siRNA transfection. Transfections and siRNA knockdown were performed as reported previously (Murray and Shewan, 2008). Briefly, siRNAs against Epac1, Epac2, the catalytic subunit of PKA (PKAc) $\alpha$, and PKAc $\beta$ were obtained from Qiagen along with All Stars negative control, nontargeting siRNA. siRNAs were targeted against the following sequences: Epac1, ATG CAC CTG TTT GGT TTC CTA; Epac2, ATC CGT GAA TGTAGT CAT TTA; PKAc $\alpha$, ATG CAT CTA ATT TAA CAT CCA; and PKAc $\beta$, TGG TAG CGT TGT ATA TTA. Both Epac1 and Epac2 or PKAc $\alpha$ and PKAc $\beta$ were used combined throughout the study. Three micrograms of each siRNA were transfected into DRG neurons using an Amaxa Nucleofector device (Lonza) using program G13. Using these methods, substantial and specific knockdown of Epac1 and Epac2 and of PKAc has been shown previously, and between 80 and $90 \%$ of neurons are transfected with these siRNAs using this technique (Murray and Shewan, 2008). For experiments in Figures $1 D$ and $4 J$ (rescue of siRNA knockdown), DRG neurons were concurrently transfected with cDNAs encoding either a green fluorescent protein (GFP)-tagged human Epac1 (Fig. $1 D$ ), kindly provided by Prof. Xiaodong Cheng (University of Texas Medical Branch, Galveston, Texas) (Qiao et al., 2002) or cyan fluorescent protein (CFP)/yellow fluorescent protein (YFP)-tagged human PKA (Zaccolo and Pozzan, 2002) along with appropriate rat siRNAs. Fluorescent cells containing the appropriate cDNA were identified using a Leica AF6000LX imaging system equipped with epifluorescence and growth cone turning assays were performed on these neurons.

Growth cone turning assays. Assays were performed according to Murray and Shewan (2008). Briefly, DRG neurons were cultured onto $13 \mathrm{~mm}$ glass coverslips coated in $10 \mu \mathrm{g} / \mathrm{ml}$ poly-L-lysine (Sigma) and further coated in $2 \mu \mathrm{g} / \mathrm{ml}$ laminin (Invitrogen). The coverslips to be assayed were transferred to a low-walled glass-bottomed $50 \mathrm{~mm}$ Petri dish (MatTek) containing BSF medium supplemented with $15 \mathrm{~mm}$ HEPES buffer (Sigma). The culture medium was overlaid with a $2-3 \mathrm{~mm}$ layer of vegetable oil, and the dish was transferred to a heated $\left(37^{\circ} \mathrm{C}\right)$ Nikon diaphot inverted microscope stage. Growth cone analysis was performed using QWin version 2.1 software. Growth cones were orientated at a $45^{\circ}$ angle and $100 \mu \mathrm{m}$ from a glass micropipette with a $1 \mu \mathrm{m}$ bore width. Reagents were ejected by an air pulse of 3 psi at $2 \mathrm{~Hz}$ and $10 \mathrm{~ms}$ duration applied to the micropipette with a Picospritzer III (Intracel). Growth cones were assayed for $30 \mathrm{~min}$, and only those that advanced $>10 \mu \mathrm{m}$ over the assay period were included in the analysis. The angle between the start and final position of the growth cone was calculated using NIH ImageJ software. The number of growth cones in each condition is indicated in parentheses in Results, and data were pooled from at least three separate experiments. When applicable, pharmacological reagents were bath applied to cultures at least $30 \mathrm{~min}$ before beginning the assays. Neurons were analyzed between 16 and $48 \mathrm{~h}$ after culture.

Immunocytochemistry. Cells were fixed for a minimum of $10 \mathrm{~min}$ at room temperature (RT) by adding an equal volume of warmed $4 \%$ paraformaldehyde (Sigma) in PBS (Sigma) to the culture medium, before washing three times in PBS. The cells were then fixed in methanol at $-20^{\circ} \mathrm{C}$ for $3 \mathrm{~min}$ before three additional washes in PBS. Cultures were immunolabeled by incubating with anti-GAP43 (diluted 1:1000; Abcam) for $2 \mathrm{~h}$ at RT. The primary antibodies were visualized using cyanine 3-conjugated anti-rabbit IgG secondary antibody (1:200). The coverslips were mounted in glycerol containing 1,4-diazobicyclo-(2,2,2)-octane (Sigma) and sealed using nail varnish.

FRET analysis. For FRET experiments to detect PKA protein activity, dissociated DRG neurons were transfected with $4 \mu \mathrm{g}$ each of RII-CFP and C-YFP cDNA constructs (kind gifts from Prof. Manuela Zaccolo, University of Glasgow, Glasgow, UK) (Zaccolo et al., 2002). Experiments investigating Epac protein activity required transfection with $8 \mu \mathrm{g}$ of a CFP-Epac-YFP cDNA construct (Ponsioen et al., 2004) (kindly provided by Dr. Kees Jalink, The Netherlands Cancer Institute, Amsterdam, The Netherlands). To detect B-Raf activity, $8 \mu \mathrm{g}$ of the Prin-B-Raf FRET construct (generously provided by Prof. Michiyuki Matsuda, University of Kyoto, Kyoto, Japan) (Teria and Matsuda, 2006) were used for neonatal neurons and $6 \mu \mathrm{g}$ for embryonic neurons. Transfection was performed using the rat neuron Nucleofection kit and program G13 on a Nucleofection II machine (Lonza). After transfection, neurons were seeded at 5000/ml on $50 \mathrm{~mm}$ glass-bottomed dishes (MatTek) coated with $10 \mu \mathrm{g} / \mathrm{ml}$ laminin.

Time-lapse recordings of transfected growth cones were simultaneously captured in CFP-, YFP-, and FRET-configured channels using a Leica AF6000LX imaging system and analyzed using Leica application software. Raw fluorescence images of $432 \mathrm{~ms}$ duration were captured every 2 min over a period of $30 \mathrm{~min}$ using a Roper Coolsnap $1392 \times 1040$ pixel camera using $3 \times 3$ binning. The Leica 11504119 Fret EX filter set was used to separate three fluorescent channels: a CFP donor channel (excitation wavelength at $427 \pm 5 \mathrm{~nm}$; emission measured at $482 \pm 15$ $\mathrm{nm}$ ), a FRET channel (excitation wavelength at $427 \pm 5 \mathrm{~nm}$; emission measured at $542 \pm 13 \mathrm{~nm}$ ) and a YFP acceptor channel (excitation wavelength of $500 \pm 6 \mathrm{~nm}$; emission measured at $542 \pm 13 \mathrm{~nm}$ ). Untransfected, donor-only and acceptor-only images were used to generate correction factors for donor crosstalk (donor emission bleeding through into the acceptor channel) and acceptor cross-excitation (acceptor excitation at the donor excitation wavelength). These correction values were used along with fluorescence measurements from regions of interest selected in the captured time-lapse channels, which had been background corrected relative to nonfluorescent regions adjacent to each captured growth cone to calculate sensitized FRET efficiency. This calculation was performed using the Leica Application Suite-Advanced Fluorescence software, which uses an algorithm defined by Wouters et al. (2001). Data are expressed as the percentage change from the initial control reading at $t_{0}$, and individual experiments were performed a minimum of three times under identical conditions.

Statistical analysis. Statistical analysis on FRET data was performed using an unpaired two-tailed Student's $t$ test. Growth cone turning assay 
data were analyzed using a Mann-Whitney $U$ test in Minitab version 5 comparing control and experimental data unless otherwise stated.

\section{Results}

Epac is necessary for growth cone attraction to a cAMP gradient, but PKA activation induces repulsion

We have shown recently that neonatal rat DRG growth cones are attracted to a gradient of the selective Epac agonist 8-MecAMP (Murray and Shewan, 2008). In the current study, we transfected embryonic DRG neurons with siRNA against Epac or PKAc and challenged them with a gradient of the cAMP analog Sp-cAMPS. We have shown previously that, with this siRNA approach, we transfect $\sim 80 \%$ of both adult and embryonic neurons and achieve substantial protein knockdown (Murray and Shewan, 2008). Neurons transfected with control siRNA demonstrated clear growth cone attraction for the Sp-cAMPS gradient with a mean turning angle (MTA) of $+13.8 \pm 2.6^{\circ}(n=11)$ (Fig. $1 A, D)$. Superimposed neurite trajectories (as shown in Fig. $1 A-C$ and in subsequent figures) provide a visual impression of neurite growth behavior and directional bias in turning assay gradients, but only the final angle of orientation of each growth cone at the end of the $30 \mathrm{~min}$ assay period was measured relative to its starting position and plotted in cumulative frequency curves (Fig. 1D). It can therefore be seen that, after $30 \mathrm{~min}, 10$ of the 12 embryonic DRG growth cones analyzed were oriented toward the SpcAMPS gradient. Knockdown of PKAc did not alter the attractive response (MTA of $+11.25 \pm 4.3^{\circ} ; n=20$ ) (Fig. $1 B, D$ ). However, knockdown of Epac significantly switched mean attraction to repulsion from the Sp-cAMPS gradient, with an average turning angle of $-9.2 \pm 5.3^{\circ}$ $(n=20)$ (Fig. 1C,D). Rescue experiments were performed by cotransfecting Epac siRNA and a cDNA encoding a GFPtagged human form of Epac1. The GFP tag allowed us to identify those growth cones expressing the human Epac and thus assay only growth cones in which Epac expression was rescued. The siRNA-induced switch from attraction to repulsion was prevented, and growth cones were again attracted to the Sp-cAMPS gradient, with an MTA of $+10.4 \pm 3.3^{\circ}$ (Fig. $1 D$ ). This demonstrates the specificity of the Epac siRNA and further confirms that Epac is necessary for growth cone attraction.

To further examine the role of PKA in cAMP-mediated axon guidance, we performed growth cone turning assays in gradients of the selective PKA activator 6-Phe-cAMP (Christensen et al., 2003). Interestingly, 6-Phe-cAMP at $5 \mathrm{~mm}$ in the pipette (5 $\mu \mathrm{M}$ at the growth cone) (Lohof et al., 1992) induced a substantial repulsive response by neonatal rat DRG growth cones showing an
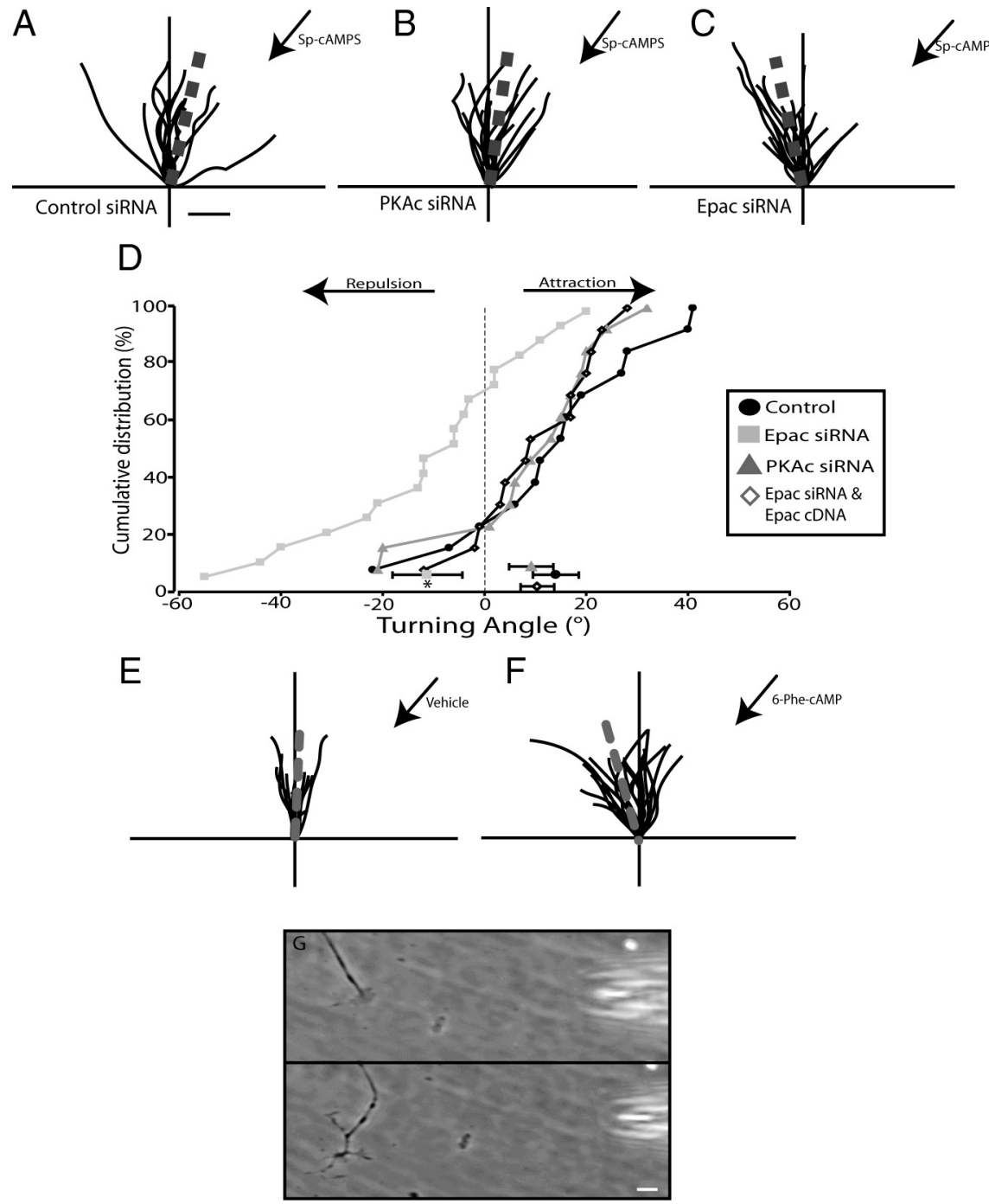

Figure 1. Epac, and not PKA, mediates growth cone attraction to a gradient of a cAMP agonist, but PKA activation induces growth cone repulsion. $\boldsymbol{A}-\boldsymbol{C}$, Superimposed neurite trajectories in embryonic growth cone turning assays. When transfected with control siRNA, growth cones are attracted to an Sp-cAMPS gradient $(\boldsymbol{A})$. Attraction is not altered when neurons are transfected with siRNA against PKAc $(\boldsymbol{B})$ but is switched to repulsion with siRNA-mediated knockdown of Epac (C). D, Cumulative frequency curves showing that the majority of control and PKAc siRNA-transfected neurons exhibit growth cone attraction to the Sp-cAMPS gradient, whereas most growth cones of Epac siRNA-transfected neurons are repelled. Transfection of human Epac1 cDNA along with Epac siRNA switches repulsion back to attraction. The turning angle of each growth cone is plotted on the abscissa against the percentage (ordinate axis) of growth cones turning to that angle or less. The mean turning angle $\pm S E$ of each group is represented by a single symbol above the abscissa. $\boldsymbol{E}, \boldsymbol{F}$, Superimposed traces of neurite trajectories in neonatal DRG growth cone turning repulsion from a gradient of the PKA agonist 6-Phe-CAMP $(\boldsymbol{F}) . \mathbf{G}$, A growth cone turning assay showing the response of a neonatal DRG growth cone at $t_{0}$ (top) and $t_{30} \mathrm{~min}$ (bottom) in a gradient of 6-Phe-CAMP. Average turning angles are indicated by dashed lines in $\boldsymbol{A}-\boldsymbol{C}, \boldsymbol{E}$, and $\boldsymbol{F}$. Scale bars: $\boldsymbol{A}, \boldsymbol{G}, 10 \mu \mathrm{m}$. ${ }^{*} p<0.05$.

MTA of $-16.2 \pm 5.9^{\circ}(n=21)$, significantly different from the vehicle control $(p<0.05)$ (Fig. $1 E-G)$. This repulsion was blocked in growth cones transfected with siRNA against PKAc but was not altered with control or Epac siRNA (supplemental Fig. $1 \mathrm{~A}$, available at www.jneurosci.org as supplemental material). Alternative concentrations of 6-Phe-cAMP at 2.5 or $10 \mu \mathrm{M}$ resulted in similar repulsive growth cone responses (supplemental Fig. $1 B$, available at www.jneurosci.org as supplemental material). To further verify that selective PKA activation causes growth cone repulsion, we used a different analog that also specifically activates PKA, 6-Mb-cAMP (Christensen et al., 2003). At concentrations of 5 and $10 \mu \mathrm{M}$ at the growth cone, a gradient of 

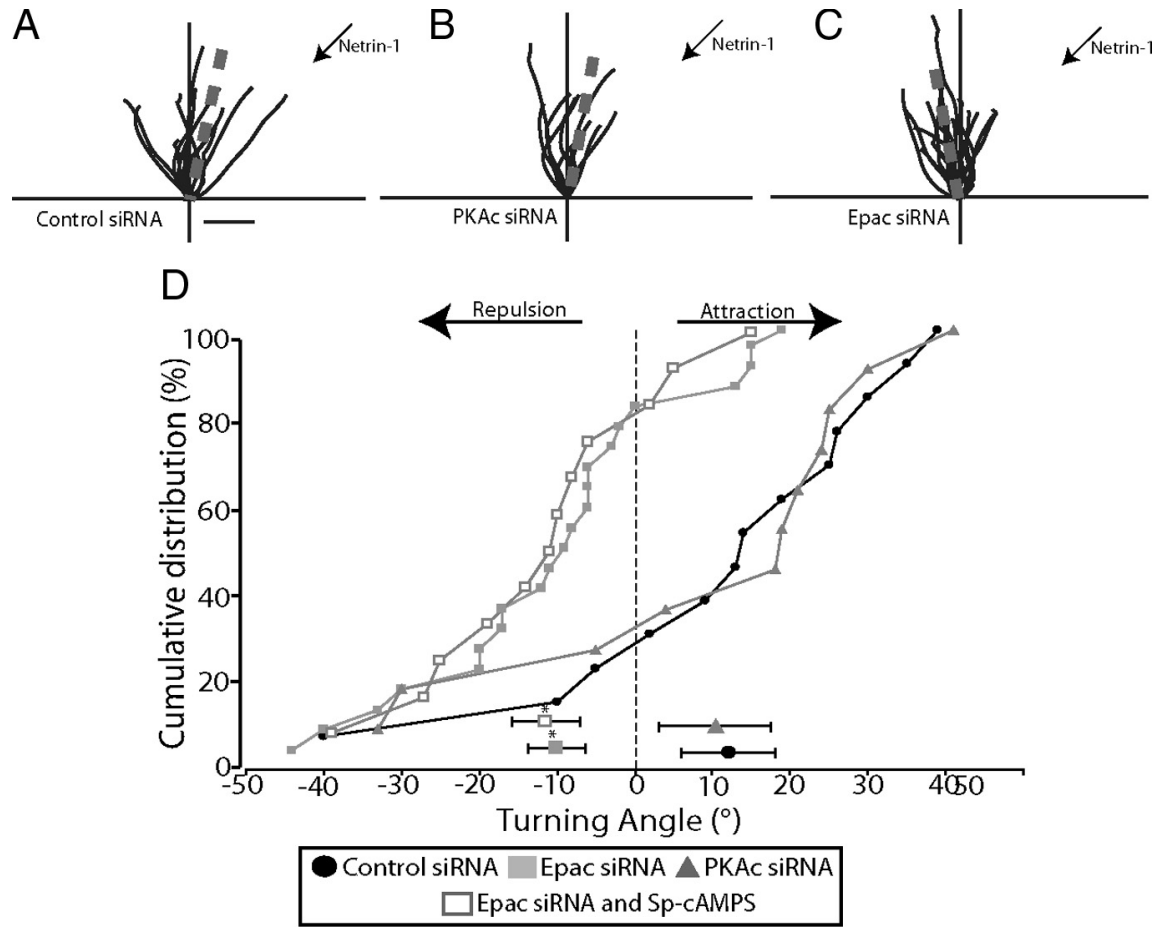

Figure 2. Epac, but not PKA, is required for embryonic DRG growth cone attraction to netrin-1. $\boldsymbol{A}-\boldsymbol{C}$, Superimposed traces of neurite trajectories in growth cone turning assays. Neurons transfected with control siRNA show growth cone attraction to a gradient of netrin-1 $(\boldsymbol{A})$. Attraction is not altered with knockdown of PKAC $(\boldsymbol{B})$ but is switched to repulsion with knockdown of Epac (C). D, Cumulative frequency curves (described in Fig. 1D) show that most growth cones of control transfected neurons are attracted to a netrin-1 gradient. Attraction is unaffected by knockdown of PKAc, but the majority of growth cones are repelled by netrin- 1 when Epac is knocked down. Average turning angles are indicated by dashed lines in $\boldsymbol{A}$-C. Scale bar: $A, 10 \mu \mathrm{m} .{ }^{*} p<0.05$.
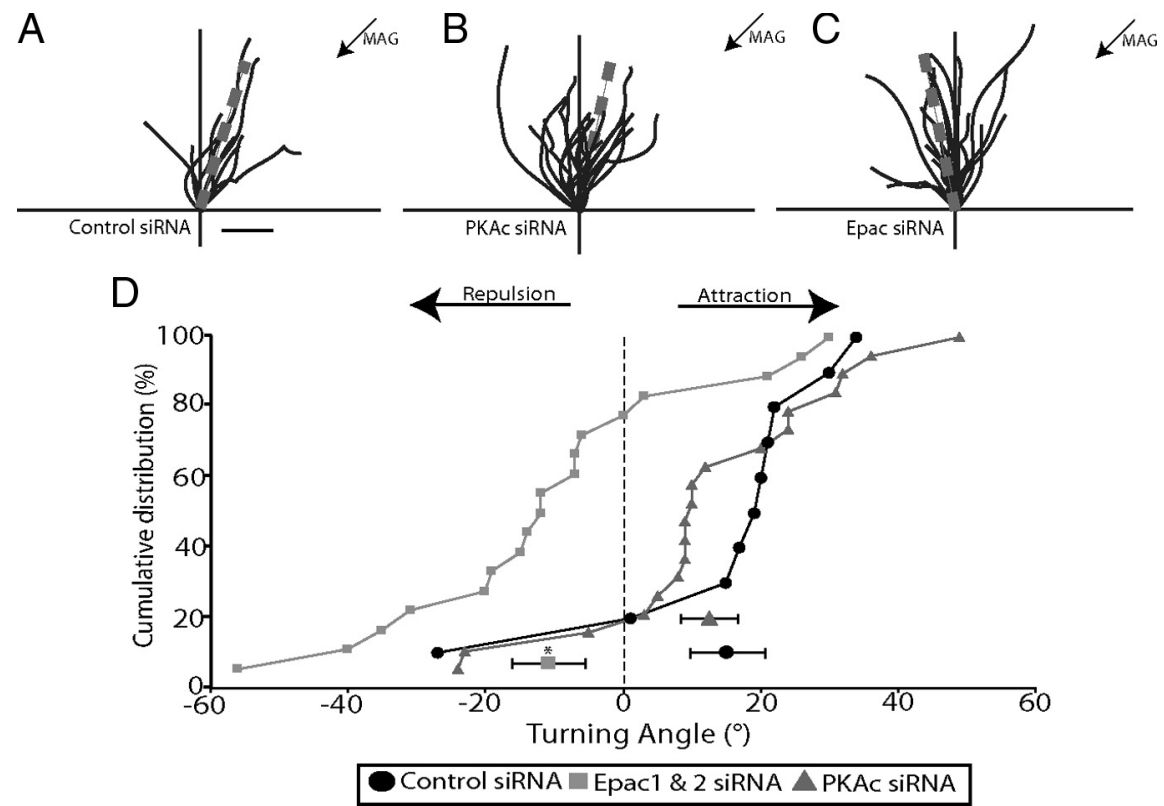

Figure 3. Epac, but not PKA, signaling mediates embryonic growth cone attraction to MAG. $\boldsymbol{A}-\boldsymbol{C}$, Superimposed neurite trajectories in turning assays show that, when transfected with control siRNA, growth cones are attracted to a MAG gradient $(\boldsymbol{A})$ and that attraction to MAG is not altered in cells transfected with PKAc siRNA $(\boldsymbol{B})$ but is switched to repulsion when Epac is knocked down (C). D, Cumulative frequency curves (described in Fig. 1D) show that the majority of growth cones of neurons transfected with control or PKAc siRNA are attracted to the MAG gradient, but most growth cones of neurons transfected with Epac siRNA are repelled by MAG. Average turning angles are indicated by dashed lines in $\boldsymbol{A}-\boldsymbol{C}$. Scale bar: $A, 10 \mu \mathrm{m} .{ }^{*} p<0.05$.
6-Mb-cAMP also induced neonatal growth cone repulsion (supplemental Fig. $1 B$, available at www.jneurosci.org as supplemental material). Furthermore, 6-PhecAMP also induced repulsion of adult growth cones, which was blocked after transfection of PKAc siRNA (supplemental Fig. $1 C$, available at www.jneurosci.org as supplemental material). Additionally, adult growth cones were attracted to a gradient of the selective Epac agonist 8-Me-cAMP, but this was blocked after transfection of Epac siRNA (supplemental Fig. $1 C$, available at www.jneurosci.org as supplemental material). Thus, asymmetric selective activation of PKA provokes repulsive turning by postnatal DRG growth cones.

Epac, but not PKA, is required for embryonic growth cone attraction to netrin-1

Growth cones have been shown to be attracted to a netrin-1 gradient when cAMP levels are high but repelled at low levels of cAMP (Ming et al., 1997; Song and Poo, 1999; Shewan et al., 2002). In our present study, we found that embryonic rat DRG growth cones showed an attractive response to a netrin- 1 gradient $(5 \mu \mathrm{g} / \mathrm{ml}$ in pipette), with an MTA of $+12.1 \pm 6.0^{\circ}$ $(n=13)$ (Fig. $2 A, D)$. This attraction was unaffected by PKAc knockdown, because the majority of growth cones continued to be attracted to the netrin-1 gradient (MTA of $+10.7 \pm 7.2^{\circ} ; n=11$ ) (Fig. $2 B, D)$. However, growth cone attraction was switched to repulsion after knockdown of Epac, with 17 of the 22 growth cones tested turning away from the netrin-1 gradient (MTA of $-10.1 \pm 3.7^{\circ}$; $n=22$ ) (Fig. $2 C, D$ ). This repulsion was not altered with bath application of $20 \mu \mathrm{M}$ Sp-cAMPS to cells transfected with Epac siRNA (MTA of $-11.4 \pm 4.3^{\circ} ; n=12$ ).

Epac, but not PKA, mediates embryonic DRG growth cone attraction to a

MAG gradient

MAG is a cAMP-dependent inhibitor of adult axon regeneration but promotes neurite outgrowth by embryonic neurons (Filbin, 2003). A MAG gradient has been shown to exhibit growth cone repulsion in vitro in Xenopus spinal neurons, but the repulsion can be switched to attraction with cAMP elevation (Song et al., 1998). We examined the response of embryonic DRG neurites to a gradient of MAG (100 $\mu \mathrm{g} / \mathrm{ml}$ in the pipette). Neurons transfected with control siRNA had a profound attractive response to MAG (MTA of $+15.2 \pm 5.5^{\circ} ; n=10$ ) (Fig. $3 A, D$ ). Inter- 
estingly, knockdown of PKAc did not alter this attractive response, because the vast majority of growth cones continued to be attracted by MAG, with an MTA of $+12.6 \pm 4.2^{\circ}(n=19)$ (Fig. $\left.3 B, D\right)$. However, transfection of Epac siRNA resulted in a switch in the attractive response to repulsion, with an MTA of $-10.8 \pm 5.3^{\circ}$ $(n=18)$ (Fig. 3C,D). Thus Epac, but not PKA, mediates cAMP-dependent attraction of embryonic rat DRG growth cones by MAG.

\section{Adult DRG growth cone repulsion by a netrin-1 gradient is blocked by Epac activation}

Growth cones with low levels of cAMP are repelled by gradients of netrin-1 (Shewan et al., 2002). When presented with a gradient of netrin-1, adult DRG growth cones demonstrated a substantial mean repulsive turn of $-17.9 \pm 5.8^{\circ}(n=17)$ (Fig. $4 A, B$ ), with 13 of the 17 growth cones turning away from the gradient. Bath application of the nonspecific agonist Sp-cAMPS $(20 \mu \mathrm{M})$ significantly switched the repulsive response to a slight attraction to the netrin-1 gradient, with an MTA of $+6.5 \pm 7.2^{\circ}(n=13)$ (Fig. $4 A, B)$. Bath application of the Epacselective agonist 8 -Me-cAMP $(2 \mu \mathrm{M})$ blocked the repulsive response of growth cones to netrin-1, showing an MTA of $+1.21 \pm 5.2^{\circ}(n=14$; data not shown $)$. However, bath application of $5 \mu \mathrm{M} 8$-MecAMP produced a mean turning angle of $+5.2 \pm 4.5^{\circ}(n=15)$ (Fig. $\left.4 A, B\right)$, similar to the response seen with application of Sp-cAMPS, and 10 of the 15 growth cones turned toward the netrin-1 gradient. In contrast, application of the selective PKA agonist 6-Phe-cAMP $(5 \mu \mathrm{M})$ did not alter the repulsive turning response of adult DRG growth cones to netrin-1, and 9 of the 11 growth cones were repelled (MTA of $-16.8 \pm 7.1^{\circ} ; n=11$ ) (Fig. $4 A, B)$.

There are no available selective antagonists of Epac and PKA. Thus, to complement the experiments using Epac and PKA agonists, we used an siRNA approach to knock down expression of Epac and PKA. Growth cones of neurons transfected with control siRNA were still strongly repelled by a netrin- 1 gradient (MTA of $-16.3 \pm 6.4^{\circ} ; n=9$ ) (Fig. $4 C, J$ ), as were growth cones of neurons in which Epac was knocked down (MTA of $-16.6 \pm 6.7^{\circ} ; n=11$ ) (Fig. $4 D, J$ ). In each case, almost all growth cones turned away from the gradient. However, knockdown of PKAc switched growth cone repulsion to attraction, with an MTA of $+10.2 \pm 2.8^{\circ}(n=13)$ (Fig. $\left.4 E, J\right)$. This was switched back to repulsion when human PKA cDNA was transfected along with the PKAc siRNA, rescuing the expression of PKAc and confirming the specificity of the siRNA knockdown $\left(\right.$ MTA of $-10.0 \pm 4.7^{\circ}$ ) (Fig. $4 J$ ). Transfection of Epac siRNA prevented bath application of $20 \mu \mathrm{M}$ Sp-cAMPS from overcom-
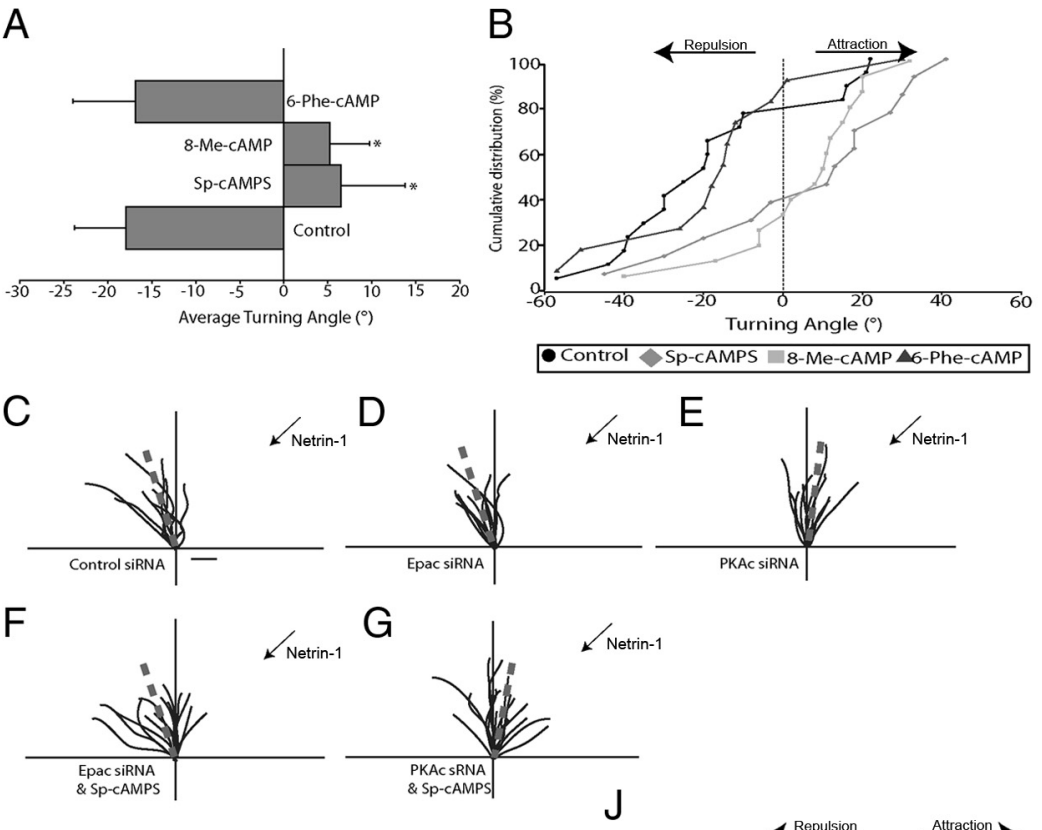

Figure 4. PKA mediates adult DRG growth cone repulsion from a netrin-1 gradient, and Epac is required for cAMP activation to switch repulsion to attraction. $A$, Bar chart of mean growth cone turning angles shows that adult growth cones - Epac agonist 8-Me-CAMP. Application of 6-Phe-CAMP has no effect on the netrin-1-induced repulsion of adult growth (he majority of adult DRG growth cones are curve to the right, indicating a switch to attraction. $\mathbf{C}-\mathbf{G}$, Superimposed neurite trajectories show that adult DRG neurons D). switch netrin-1-induced repulsion to attraction $(\boldsymbol{F})$, but neurites transfected with PKAc siRNA and treated with Sp-cAMPS are attracted to netrin-1 $(\boldsymbol{G}) . \boldsymbol{H}, \boldsymbol{I}$, Representative images of growth cones in turning assays in a netrin-1 gradient after transfection with control siRNA $(\boldsymbol{H})$ or PKAc siRNA $(\boldsymbol{I})$. Top panels show positions at $t_{0}$ and bottom panels at $t_{30}$ min. by a netrin-1 gradient, even after Sp-cAMPS application, but most growth cones of neurons transfected with PKAc siRNA are attracted to netrin-1. Attraction is switched back to repulsion with concurrent transfection of human PKA CDNA and PKAC siRNA. Dashed lines in $\boldsymbol{C}-\boldsymbol{G}$ represent mean turning angles. Scale bars: $\boldsymbol{C}, \boldsymbol{H}, 10 \mu \mathrm{m} .{ }^{*} p<0.05$.

ing repulsion (MTA of $-17.5 \pm 5.5^{\circ} ; n=14$ ) (Fig. $4 F, J$ ), but application of Sp-cAMPS to neurons transfected with PKAc siRNA did switch growth cone repulsion to attraction in the netrin-1 gradient (MTA of $+11.4 \pm 4.8^{\circ} ; n=16$ ) (Fig. $4 G, J$ ). These data demonstrate that attractive responses to netrin-1 are mediated by Epac whereas repulsion is mediated by PKA and that Epac is required for Sp-cAMPS to overcome growth cone repulsion.

\section{Adult DRG growth cone repulsion by MAG is mediated by PKA, and Epac is required to switch repulsion to attraction on activation of cAMP}

When challenged with a gradient of MAG, adult DRG growth cones showed a substantial repulsive response (MTA of $-19.8 \pm$ $5.4^{\circ}$ ), with 20 of 24 growth cones repelled (Fig. $5 A, E$ ). Bath ap- 

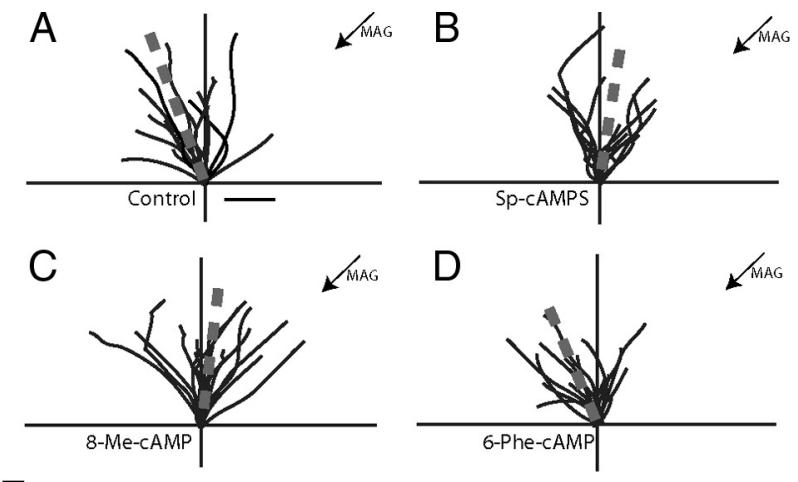

E



Control Sp-cAMPS 8-Me-cAMP A 6-Phe-cAMP

$\mathrm{F}$
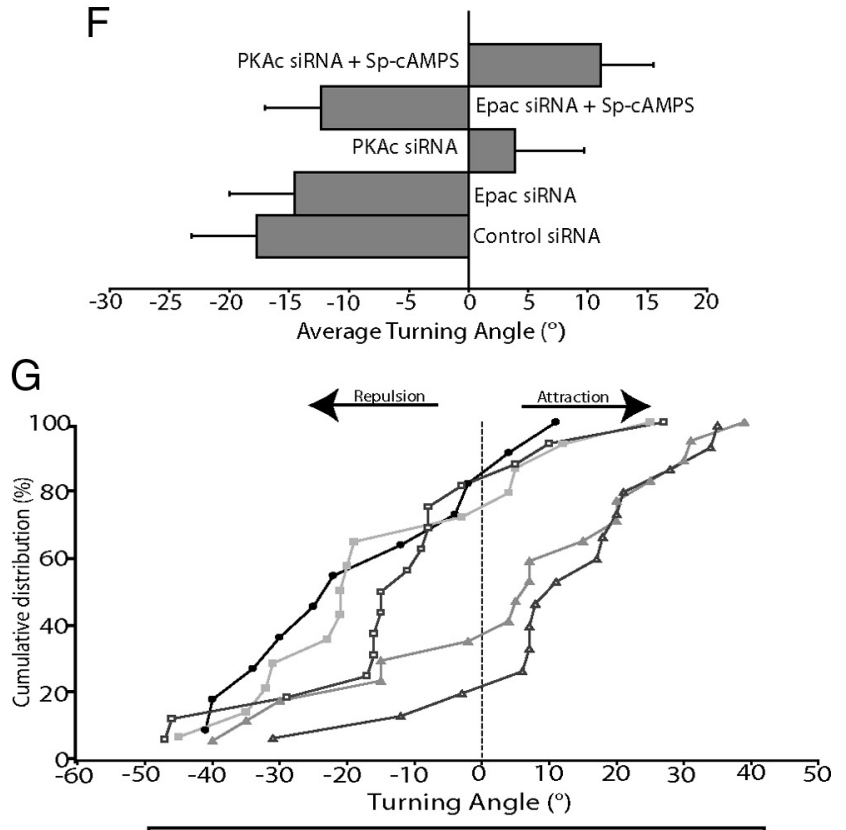

Control siRNA Epac siRNA $\triangle$ PKAc siRNA

$\triangle$ PKAC SIRNA \& Sp-CAMPS $\square$ Epac SiRNA \& SP-CAMPS

Figure 5. PKA mediates adult DRG growth cone repulsion from a MAG gradient, and Epac is required for CAMP activation to switch repulsion to attraction. $\boldsymbol{A}-\boldsymbol{D}$, Superimposed neurite trajectories in turning assays show that adult growth cones are repelled by a MAG gradient $(\boldsymbol{A})$. Repulsion is switched to attraction on application of Sp-CAMPS (B). Application of 8-Me-cAMP also overcomes the repulsion $(\boldsymbol{C})$, but growth cones are still repelled by MAG on application of 6-Phe-CAMP (D). E, Cumulative frequency curves (described in Fig. 1D) show that the majority of growth cones are repelled by MAG under control conditions or on application of 6-Phe-CAMP, but a clear shift to the right (attraction) occurs when CAMP or Epac is activated. $\boldsymbol{F}$, Bar chart shows mean growth cone turning angles of siRNA-transfected adult DRG neurons in a gradient of MAG. Transfection with a control siRNA does not alter growth cone repulsion from the MAG gradient, and siRNA-meditated knockdown of Epac is similarly ineffective. Growth cone repulsion is blocked with knockdown of PKAc. Sp-cAMPS cannot switch repulsion to attraction when Epac is knocked down but can induce attraction when PKAc is knocked down. G, Cumulative plication of $20 \mu \mathrm{M}$ Sp-cAMPS switched the MTA to $+8.3 \pm 6.4^{\circ}$, and only 2 of 14 growth cones were repelled by MAG (Fig. $5 B, E$ ). Bath application of the Epac agonist 8-Me-cAMP $(2 \mu \mathrm{M})$ had a similar effect, inducing attraction of 13 of 17 growth cones, with an average turn of $+9.6 \pm 6.1^{\circ}$ (Fig. $5 C, E$ ). However, application of $5 \mu \mathrm{M}$ 6-Phe-cAMP did not alter the repulsive response (MTA of $-21.8 \pm 5.5^{\circ}$ ) (Fig. $5 D, E$ ). 6-Phe-cAMP at $10 \mu \mathrm{M}$ was also ineffective in altering growth cone repulsion (MTA of $-13.8 \pm$ $5.7^{\circ} ; n=9$; data not shown).

Growth cones transfected with control siRNA showed an MTA of $-17.2 \pm 5.5^{\circ}(n=11)$ (Fig. $\left.5 F, G\right)$, similar to that seen without siRNA transfection. Epac knockdown did not alter the repulsive response (MTA of $-14.6 \pm 5.4^{\circ} ; n=14$ ) (Fig. $\left.5 F, G\right)$. Knockdown of PKAc, however, significantly altered the turning angle to a more neutral response, with an MTA of $+3.9 \pm 5.8^{\circ}$ $(n=17)$ (Fig. $5 F, G)$. Moreover, in Epac siRNA-transfected neurons, bath application of Sp-cAMPS no longer switched growth cone repulsion to attraction, showing an MTA of $-17.9 \pm 5.5^{\circ}$ $(n=14)$ (Fig. $5 F, G)$. In contrast, PKAc siRNA-transfected neuronal growth cones showed a robust attraction to the MAG gradient, displaying an MTA of $+11.1 \pm 4.5^{\circ}(n=15)$ (Fig. $5 F, G)$. These data strongly indicate that embryonic DRG growth cone attraction to a MAG gradient is mediated by Epac whereas repulsion of adult growth cones is mediated by PKA and that Epac is required for cAMP activation to overcome growth cone repulsion.

\section{FRET analysis reveals distinct modes of cAMP signaling in embryonic and neonatal DRG neurons}

The data presented above indicate that Epac mediates growth cone attractive responses to cAMP-dependent axon guidance cues and PKA mediates repulsion. This would suggest a switch in the mode of cAMP signaling in developing neurons, because embryonic neuronal growth cones are attracted to cAMP-dependent guidance cues such as netrin-1 and MAG, whereas postnatal growth cones are repelled. To test this, we have established the FRET technique in cultured DRG neurons to measure protein activity in live growth cones after bath application of cAMP agonists or netrin-1. We found that selective Epac and PKA agonists in the concentration range used in growth cone turning assays distinctly activated Epac and PKA, respectively, to similar extents in both embryonic (Fig. 6A-D) and neonatal (data not shown) DRG neurons, confirming the specificity of these agonists. Intriguingly, we further found that bath application of netrin-1 at the concentration $(5 \mathrm{ng} / \mathrm{ml})$ detected by growth cones in turning assays exhibited an increase in Epac, but not PKA, activity in embryonic neurons but, conversely, increased PKA, but not Epac, activity in neonatal neurons (Fig. 6E-H). Because netrin-1 and MAG in growth cone turning assays attract embryonic growth cones (Figs. 2, 3) but are repellent to both neonatal (supplemental Fig. 2, available at www.jneurosci.org as supplemental material) and adult (Figs. 4, 5) growth cones, these FRET data support our turning assay work in suggesting that Epac mediates attraction and PKA mediates repulsion. Moreover, the FRET data imply that the switch in developing DRG growth cone response to gradients of cAMP-dependent axon guidance cues is the result

$\leftarrow$

frequency curves show that most growth cones of control and Epac siRNA-transfected neurons are repelled by MAG, even when Sp-CAMPS is applied. When PKAc is knocked down, the majority of growth cones are attracted to $M A G$, and all but three are attracted. Average turning angles are indicated by dashed lines in $\boldsymbol{A}-\boldsymbol{D}$. Scale bar: $\boldsymbol{A}, 10 \mu \mathrm{m}$. ${ }^{*} p<0.05$. 
of a switch in the mode of cAMP signaling from Epac-mediated to PKA-mediated mechanisms.

Epac and PKA have differential effects on calcium and B-Raf signaling

Our data imply that Epac and PKA regulate distinct signaling pathways in DRG growth cones to elicit growth cone attraction and repulsion, respectively. This suggests that Epac and PKA either have distinct downstream targets or differentially mediate common downstream signaling molecules. $\mathrm{Ca}^{2+}$ has been shown to regulate both cAMP- and Epac-mediated cell signaling. To test whether Epac provokes a physiological response in DRG growth cones, we performed calcium imaging studies in embryonic DRG neurons after application of 2 $\mu \mathrm{M} 8$-Me-cAMP in neurons that had been transfected with control siRNA or Epac siRNA. In control neurons, application of 8-Me-cAMP substantially raised growth cone $\mathrm{Ca}^{2+}$ levels, and this activation was significantly greater than the small rise seen when Epac was knocked down (Fig. $7 A, C, D)$. Netrin-1 has been shown to induce calcium elevation in growth cones (Hong et al., 2000), and studies have shown that reducing the netrin- $1 \mathrm{Ca}^{2+}$ response by blocking either $\mathrm{Ca}^{2+}$ influx or its release from intracellular stores is sufficient to switch attraction to repulsion (Hong et al., 2000). We found that application of $5 \mathrm{ng} / \mathrm{ml}$ netrin-1 raised $\mathrm{Ca}^{2+}$ levels in embryonic DRG growth cones. This $\mathrm{Ca}^{2+}$ elevation was absent in growth cones transfected with Epac siRNA (Fig. 7B,E,F). Thus, Epac activation induces a physiological response within DRG growth cones by increasing $\left[\mathrm{Ca}^{2+}\right]_{\mathrm{i}}$, and Epac is required for netrin-1induced $\mathrm{Ca}^{2+}$ elevation.

B-Raf can be regulated by both Epac and PKA. B-Raf promotes neurite outgrowth, and, when knocked out, DRG axon guidance in development is perturbed (Zhong et al., 2007). We transfected embryonic and postnatal DRG neurons with a B-Raf FRET construct to test whether B-Raf might be differentially regulated by Epac and PKA. We found that, in both embryonic and neonatal neurons, 8-Me-cAMP activates B-Raf (Fig. 7G,H) but 6-Phe-cAMP induces decreased B-Raf activity (Fig. $7 G, H$ ). Interestingly, netrin-1 only activated B-Raf in embryonic growth cones but had no effect on B-Raf in neonatal neurons (Fig. 7G,H). Thus, the differential activation of B-Raf by Epac- and PKA-mediated cell signaling may underlie a mechanism by which cAMP can mediate different responses in growth cones.
A

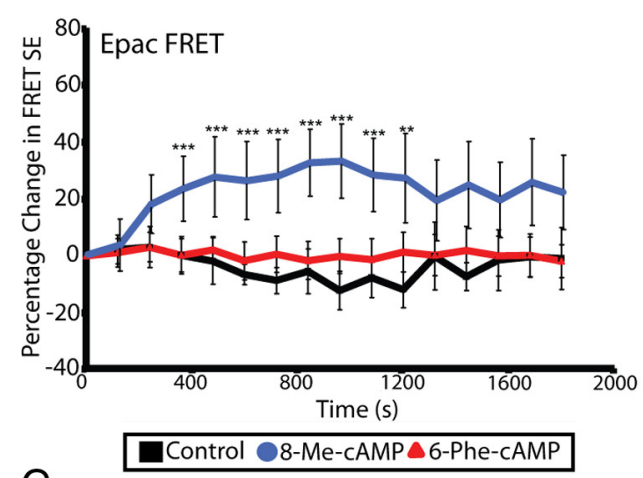

B
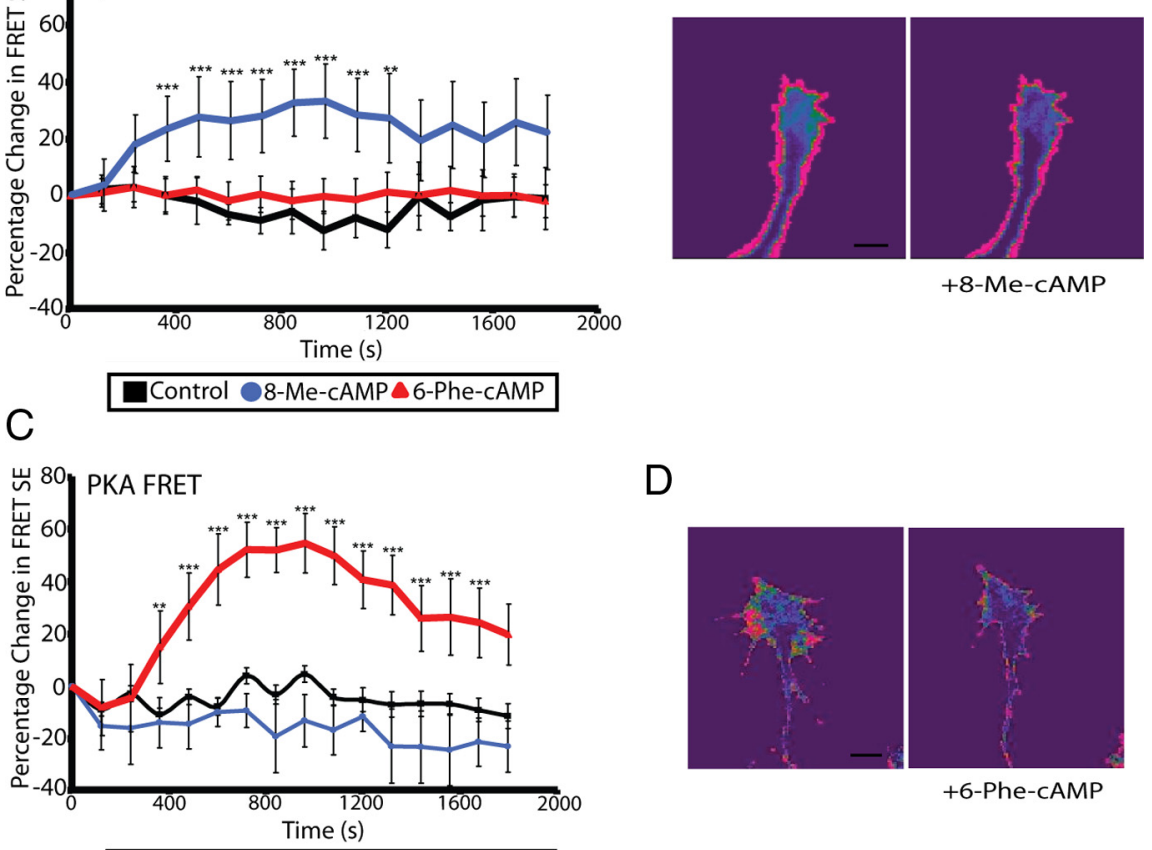

D

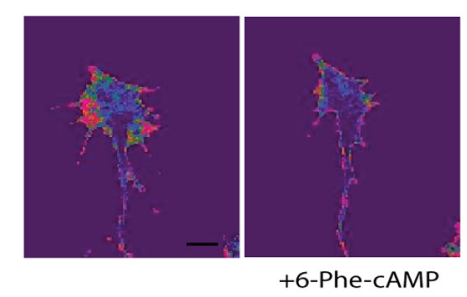

E

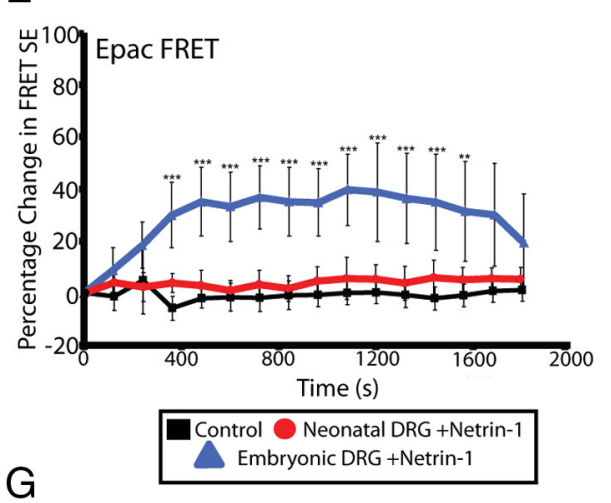

F

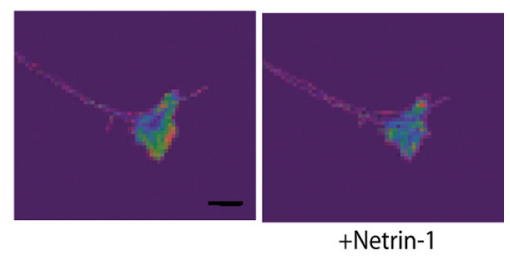

Embryonic DRG

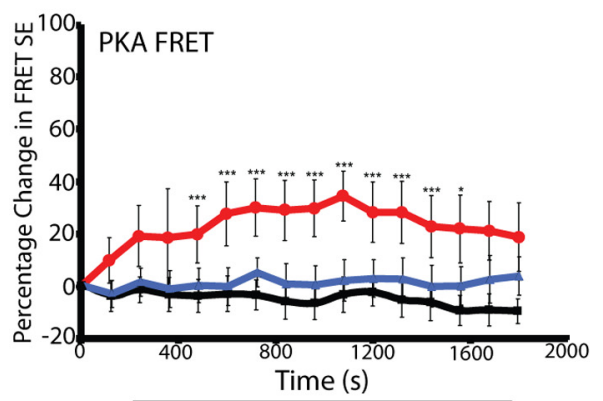

$\mathrm{H}$
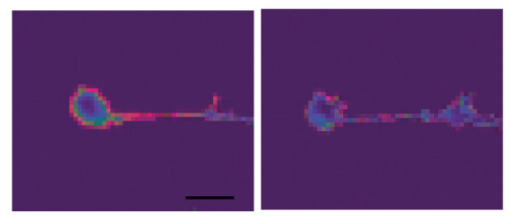

Neonatal DRG

Control Neonatal DRG + Netrin-1 Embryonic DRG + Netrin-1

Figure 6. FRET analysis confirms the specificity of selective Epac and PKA agonists and shows that netrin-1 activates Epac in embryonic neurons but PKA in postnatal neurons. $\boldsymbol{A}, \boldsymbol{B}$, Embryonic DRG neurons transfected with an Epac FRET sensor show an increase in growth cone Epac activation on application of $2 \mu \mathrm{m} 8$-Me-cAMP but no change in Epac activity after application of $5 \mu \mathrm{M}$ 6-Phe-CAMP or a vehicle control $(\boldsymbol{A}) . \boldsymbol{C}, \boldsymbol{D}$, Embryonic DRG neurons transfected with PKA FRET constructs show an increase in growth cone PKA activation on application of 6-Phe-CAMP but no change in PKA activity after application of 8-Me-cAMP or a vehicle control (C). $\boldsymbol{E}-\boldsymbol{H}$, Epac, but not PKA, activation is detected in embryonic DRG growth cones on application of $5 \mu \mathrm{g} / \mathrm{ml}$ netrin-1 $(\boldsymbol{E}, \boldsymbol{F})$, but PKA and not Epac is activated when netrin-1 is applied to neonatal growth cones $(\boldsymbol{G}, \boldsymbol{H}) . \boldsymbol{B}, \boldsymbol{D}, \boldsymbol{F}, \boldsymbol{H}$, Increased protein activity is represented by darker blue/purple colors within the growth cone (right panels; 5 min after bath application of selective agonists or netrin-1) compared with brighter blue/green colors before application (left panels). Scale bars, $10 \mu \mathrm{m}$. ${ }^{*} p<0.05$, ${ }^{* *} p<0.01,{ }^{* * *} p<0.001$. 
A

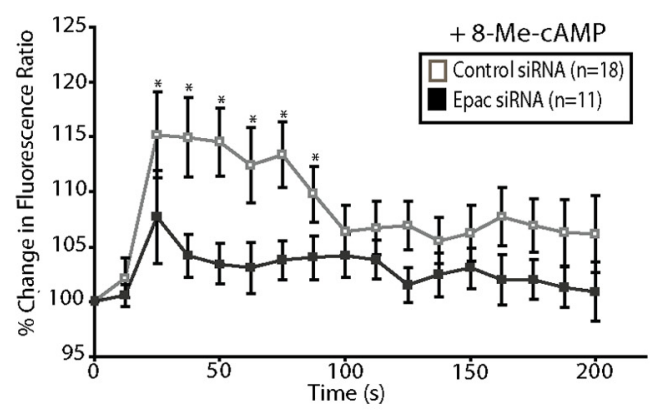

C Control siRNA - 8-Me-cAMP
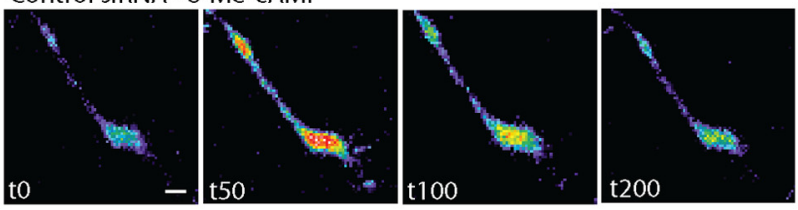

E



G

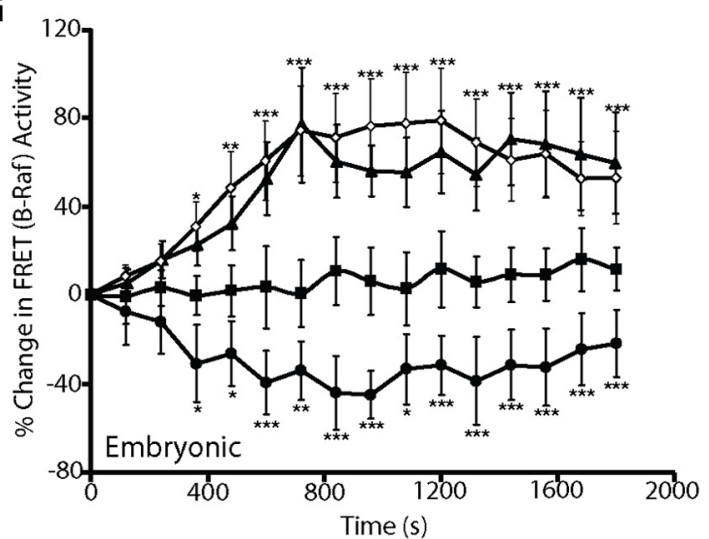

B

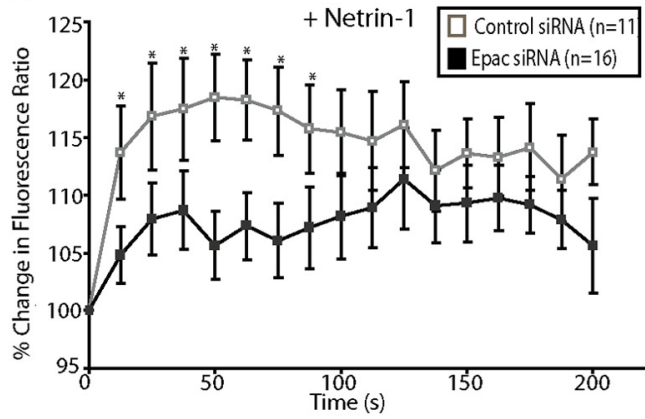

Epac siRNA - 8-Me-cAMP
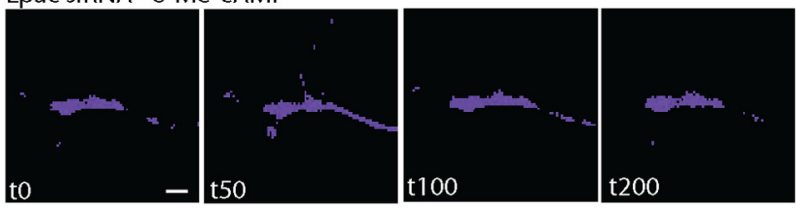

$\mathrm{F}$
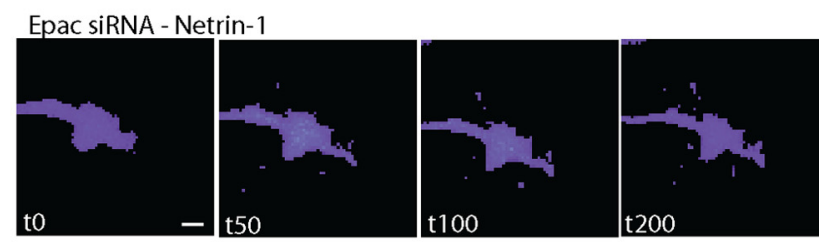

$\mathrm{H}$

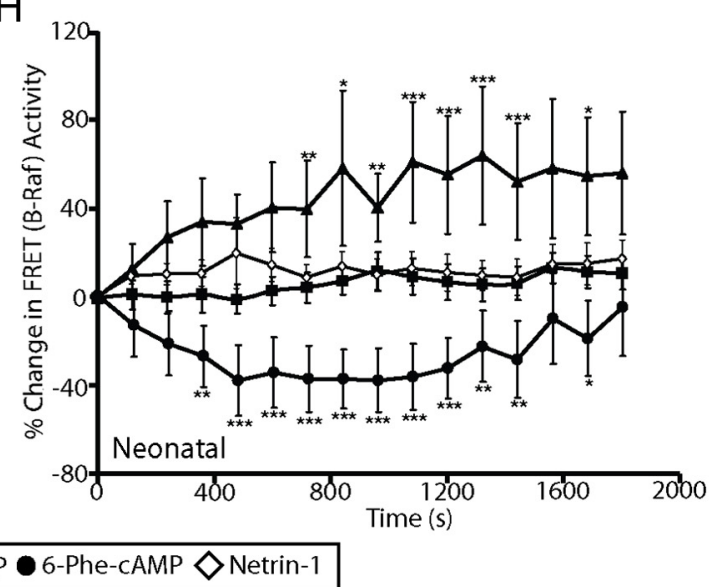

Figure 7. Growth cone calcium imaging and FRET analysis of B-Raf protein activity. $\boldsymbol{A}, \boldsymbol{C}, \boldsymbol{D}$, Bath application of 8-Me-CAMP raises calcium in embryonic DRG growth cones ( $\boldsymbol{A}, \boldsymbol{C})$ but not in neurons transfected with Epac siRNA $(\boldsymbol{A}, \boldsymbol{D}) . \boldsymbol{B}, \boldsymbol{E}, \boldsymbol{F}$, Application of netrin-1 also increases calcium in embryonic growth cones $(\boldsymbol{B}, \boldsymbol{E})$, but this is significantly reduced when Epac is knocked down $(\boldsymbol{B}, \boldsymbol{F})$. Number of growth cones analyzed in each condition shown in parentheses in legends of $\boldsymbol{A}$ and $\boldsymbol{B}$. $\boldsymbol{C}-\boldsymbol{F}$, Representative examples of fluorescence ratio images of fura-2-loaded growth cones with application of 8-Me-CAMP $(\boldsymbol{C}, \boldsymbol{D})$ or netrin-1 $(\boldsymbol{E}, \boldsymbol{F})$ in neurons transfected with control $(\boldsymbol{C}, \boldsymbol{E})$ or Epac siRNA $(\boldsymbol{D}, \boldsymbol{F})$. $\boldsymbol{G}, \boldsymbol{H}$, FRET analysis of B-Raf protein activity in embryonic $(\boldsymbol{G})$ and neonatal $(\boldsymbol{H})$ DRG growth cones. G, 8-Me-cAMP or netrin-1 application increases B-Raf activity in embryonic growth cones, but 6-Phe-cAMP decreases B-Raf activity. $\boldsymbol{H}$, B-Raf activity is also increased by 8-Me-cAMP and decreased by 6-Phe-cAMP in neonatal growth cones, but netrin-1 no longer affects B-Raf activity. Scale bars: $\mathbf{C}-\boldsymbol{F}, 5 \mu \mathrm{m} .{ }^{*} p<0.05,{ }^{* *} p<0.01,{ }^{* * *} p<0.001$.

\section{Discussion}

cAMP transduces growth cone responses to a number of axon guidance cues, with high cAMP levels mediating growth cone attraction and low levels mediating repulsion (Song and Poo, 1999). In Xenopus, growth cones switch their response from attraction to repulsion in a gradient of netrin-1 as retinal axons extend from the retina to the tectum. Endogenous growth cone cAMP levels decline over this same period of development, and the repulsion can be reversed to attraction by artificially raising cAMP levels using cell-permeable agonists (Shewan et al., 2002). Developing mammalian DRG neurons also undergo a decline in endogenous cAMP levels (Cai et al., 2001), thought to underlie the switch from MAG acting as an attractive, growth-promoting cue for embryonic neurons to becoming a repulsive, growthinhibiting influence on postnatal neurons. This switch has been reported to occur at postnatal day 3 in the rat (Johnson et al., 1989; Mukhopadhyay et al., 1994; DeBellard et al., 1996). We also found that MAG, and indeed netrin-1, induced growth cone repulsion in neonatal DRG neurons (supplemental Fig. 2, available at www.jneurosci.org as supplemental material). We did not attempt to identify the specific time point that the switch from growth cone attraction to repulsion occurs, and the growth cone turning assay is very different from neurite outgrowth assays on MAG-expressing cells. In the latter case, neurons are plated on cell monolayers and thus exposed to surrounding, unknown concentrations of MAG from the moment of plating. In growth cone 
turning assays, neurons have already generated long neurites before being confronted with a MAG gradient of known concentration, shown previously to be effective in growth cone turning (Wong et al., 2002), on only one side of the growth cone, and only neurites that continue to advance are assayed. Thus, the concentration of MAG detected by the growth cone is likely to be very different from that confronted by neurons plated on MAGexpressing cells in previous studies, and the mode of application of MAG is very different. Nevertheless, most of the neurons in our study were prepared from animals at least postnatal day 2 in age and analyzed 16-48 h later, and thus our observations that neonatal neurons become repelled by MAG coincide very closely with the developmental time point at which MAG has been shown previously to become inhibitory to rat DRG neurite outgrowth.

The mechanisms by which cAMP mediates these polarized responses are unknown, but it seems unlikely that only PKA-mediated signaling is involved. Moore and Kennedy (2006) found that PKA activation is not required for rat commissural axon attraction to netrin-1 and that mechanisms underlying netrin-1 attraction for growth cones are distinct from mechanisms required for switching attraction to repulsion. The identification of Epac, the guanine nucleotide exchange factor for Rap1 (de Rooij et al., 1998; Kawasaki et al., 1998), led us to assess the relative contributions of Epac and PKA in cAMP-dependent rat DRG growth cone guidance. Our results imply that growth cone attraction and repulsion are distinctly regulated by Epac and PKA, respectively, and that the developmental switch in response to cAMP-dependent guidance cues from attraction to repulsion occurs because cAMP switches from activating Epac to signaling through PKA (Fig. 8).

By what mechanisms might Epac and PKA signaling be differentially regulated? A number of studies have shown that Epac and PKA are activated at different cAMP concentrations. PKA responds to relatively low levels of cAMP and is activated in the low to mid nanomolar range (Dostmann and Taylor, 1991). FRET studies in A431 cells revealed that cellular PKA activity becomes saturated at relatively low cAMP concentrations but Epac activation requires much higher cAMP levels $(\sim 10-20 \mu \mathrm{M})$, and declining cAMP levels result in a drop in Epac FRET activity long before a decline in PKA activity is detected (Ponsioen et al., 2004). Biochemical analysis has shown that cAMP displays an almost 500-fold lower affinity for Epac than PKA (Enserink et al., 2002; Rehmann et al., 2003). Perhaps, then, when intracellular levels of cAMP are high, as in embryonic neurons, Epac is activated and mediates growth cone attraction to cAMP-dependent guidance cues. When cAMP levels decline, as in postnatal neurons, Epac may not be activated and CAMP signaling is instead mediated by PKA, inducing growth cone repulsion (Fig. 8). We propose that, when Epac is active, its chemoattractive effects outweigh the repulsive effects mediated by PKA because Epac activation, like activation of cAMP itself, switches growth cone repulsion to attraction in gradients of MAG and netrin-1, and growth cones are attracted to a gradient of Epac agonist, as they are to a cAMP gradient (Murray and Shewan, 2008). Knocking down PKA does not perturb attraction to cAMP, MAG, or netrin-1, but knocking down Epac results in repulsion, when PKA activity is presumably prevalent over Epac.

The dominance of Epac or PKA activity is important because they may differentially regulate downstream signaling molecules. In this study, FRET analysis revealed that Epac activates B-Raf but PKA inhibits it (Fig. 7). Moreover, netrin-1 only activates B-Raf in embryonic neurons, in which it activates Epac as cAMP levels are high. In postnatal neurons in which cAMP levels are low, netrin-1 does not activate Epac and does not activate B-Raf. Rather, netrin-1 activates PKA, which inhibits B-Raf. Because B-Raf activity is important for DRG neuronal development, neurite outgrowth in vitro, and axon guidance in vivo (Zhong et al., 2007), its differential regulation by PKA and Epac may mediate the different effects PKA and Epac have on growth cone dynamics. If this were the case, it seems likely that siRNA against B-Raf would block the attractive responses to gradients of netrin-1 or the Epac agonist.

A growing body of evidence shows that Epac also regulates intracellular calcium dynamics (Holz et al., 2006). $\mathrm{Ca}^{2+}$ signaling is required for growth cone responses to both MAG and netrin-1 (Ming et al., 1997; Han et al., 2007), and a gradient of $\mathrm{Ca}^{2+}$ elevation occurs in growth cones toward the direction of netrin-1 detection (Hong et al., 2000). Calcium calmodulin protein kinase II (CaMKII) and calcineurin have been implicated in growth cone attraction and repulsion, respectively (Wen et al., 2004), and Epac has been shown to modulate CaMKII activity in rat cardiac myocytes (Pereira et al., 2007). Calcium-induced calcium release (CICR) is required for growth cone attraction to netrin-1 (Hong et al., 2000), and ryanodine receptor activation can induce attractive responses by growth cones (Ooashi et al., 2005; Tojima 
et al., 2007). The Epac agonist 8-Me-cAMP induces phosphorylation of ryanodine receptors (Pereira et al., 2007), and Epac can stimulate CICR (Kang et al., 2003). Furthermore, vesicular exocytosis has been shown to mediate growth cone attraction to a MAG gradient (Tojima et al., 2007) but is not required for growth cone repulsion. Epac has been implicated in exocytosis in a number of different cell types (Seino and Shibasaki, 2005) and can facilitate neurotransmitter release from vesicles (Zhong and Zucker, 2005). In our present study, we found that application of the Epac agonist 8-Me-cAMP evoked a calcium response in embryonic DRG neurons, but not when Epac was knocked down. Moreover, the calcium response induced by netrin-1 was abolished when Epac was knocked down. If, as our results suggest, the downstream effect of netrin-1-induced Epac activation is mediated by B-Raf activation, siRNA against B-Raf might also be expected to block the netrin-1 or Epac agonist-induced rise in calcium release.

The identification of Epac as a distinct protein directly activated by cAMP has opened up new avenues of research to study the mechanisms underlying axon growth, guidance, and regeneration. Numerous cellular functions previously attributed to PKA are now being accredited to Epac (Bos, 2003). Recent gains in knowledge have revealed why previous axon guidance studies that implicated PKA in both growth cone attraction and repulsion should be reconsidered. Many studies used the antagonist Rp-cAMPS to reduce cAMP activity, switching growth cone attraction for gradients of cAMP-dependent cues to repulsion (Ming et al., 1997; Song et al., 1998). However, it is now known that Rp-cAMPS binds to both PKA and Epac, and therefore this approach cannot discriminate between the two proteins activated by cAMP (Rehmann et al., 2003). Other studies have found that application of pharmacological inhibitors, such as H89 (N-[2( $p$-bromo-cinnamylamino)-ethyl]-5-isoquinoline-sulfonamide $2 \mathrm{HCl})$ and $\mathrm{KT} 5720 \quad[(8 R, 9 S, 11 S)-(-)-9$-hydroxy-9hexoxycarbonyl-8-methyl-2,3,9,10-tetrahydro-8,11-epoxy- $1 \mathrm{H}, 8 \mathrm{H}$, $11 \mathrm{H}$-2,7b,11a-triazadibenzo[a,g]cycloocta[cde]trinden-1-one], prevents growth cone attraction to gradients of cAMP agonist, MAG, or netrin-1 (Ming et al., 1997; Song et al., 1998; Guirland et al., 2003; Munck et al., 2004; Ooashi et al., 2005). Indeed, we also found that $200 \mathrm{nM}$ KT5720 reduced the attraction for netrin-1 and Sp-cAMPS gradients by embryonic rat DRG growth cones, although it did not switch attraction to repulsion (supplemental Fig. 3, available at www.jneurosci.org as supplemental material). These PKA inhibitors antagonize the ATP-binding site on the catalytic subunit of PKA (Kase et al., 1987; Engh et al., 1996). However, KT5720 and H89 have potent antagonistic effects on many other key cell signaling molecules involved in growth cone dynamics. H89 inhibits protein kinase B, protein kinase $C$, and $\beta 2$-adrenergic receptors and alters cell excitability by affecting $\mathrm{Na}^{+}$and $\mathrm{K}^{+}$channel conductance (Penn et al., 1999; Davies et al., 2000; Murray, 2008), whereas both H89 and KT5720 affect Rho kinase, mitogen-activated protein kinase, and $\mathrm{Ca}^{2+}$ activity.

Thus, the use of nonspecific antagonists that affect a wide range of cell signaling molecules other than PKA, including many that regulate signaling mechanisms downstream of Epac, mean that events in the growth cone previously attributed to PKA should be reconsidered and clarified by using more selective and specific approaches. Here, we provide the first evidence suggesting that cAMP differentially mediates growth cone attraction and repulsion by distinctly activating Epac or PKA, respectively. We propose that, as cAMP levels decline in development, growth cones that were attracted to cAMP-dependent guidance cues be- come repelled as cAMP switches from signaling predominantly through Epac to a PKA-regulated pathway. We suggest that this leads to differential activation of B-Raf and thus the distinct regulation of downstream signaling pathways. Additional investigation into these mechanisms will enhance understanding of how the nervous system is wired during development but will also pinpoint additional targets to promote axon regeneration. Elevation of cAMP activity can enhance regeneration of injured axons in the mammalian spinal cord (Neumann et al., 2002; Qiu et al., 2002), but cAMP is expressed in all cells and regulates many important functions. The results reported here, along with our previous study demonstrating that Epac activation promotes regeneration of adult DRG neurons in vitro (Murray and Shewan, 2008), suggest that Epac and proteins farther downstream may represent more effective and appropriate targets for promoting axon regeneration in vivo.

\section{References}

Bos JL (2003) Epac: a new cAMP target and new avenues in cAMP research. Nat Rev Mol Cell Biol 4:733-738.

Cai D, Qiu J, Cao Z, McAtee M, Bregman BS, Filbin MT (2001) Neuronal cyclic AMP controls the developmental loss in ability of axons to regenerate. J Neurosci 21:4731-4739.

Christensen AE, Selheim F, de Rooij J, Dremier S, Schwede F, Dao KK, Martinez A, Maenhaut C, Bos JL, Genieser HG, Døskeland SO (2003) cAMP analog mapping of Epacl and cAMP kinase. Discriminating analogs demonstrate that Epac and cAMP kinase act synergistically to promote PC-12 cell neurite extension. J Biol Chem 278:35394-35402.

Davies SP, Reddy H, Caivano M, Cohen P (2000) Specificity and mechanism of action of some commonly used protein kinase inhibitors. Biochem J 351:95-105.

DeBellard ME, Tang S, Mukhopadhyay G, Shen YJ, Filbin MT (1996) Myelin-associated glycoprotein inhibits axonal regeneration from a variety of neurons via interaction with sialoglycoprotein. Mol Cell Neurosci 7:89-101.

de Rooij J, Zwartkruis FJ, Verheijen MH, Cool RH, Nijman SM, Wittinghofer A, Bos JL (1998) Epac is a Rap1 guanine-nucleotide-exchange factor directly activated by cAMP. Nature 396:474-477.

Dostmann WR, Taylor SS (1991) Identifying the molecular switches that determine whether (Rp)-cAMPS functions as an antagonist or an agonist in the activation of cAMP-dependent protein kinase. Biochemistry 30:8710-8716.

Engh RA, Girod A, Kinzel V, Huber R, Bossemeyer D (1996) Crystal structures of catalytic subunit of cAMP-dependent protein kinase in complex with isoquinolinesulfonyl protein kinase inhibitors H7, H8, and H89. Structural implications for selectivity. J Biol Chem 271:26157-26164.

Enserink JM, Christensen AE, de Rooij J, van Triest M, Schwede F, Genieser HG, Døskeland SO, Blank JL, Bos JL (2002) A novel Epac specific cAMP analogue demonstrates independent regulation of Rap1 and Erk. Nat Cell Biol 4:901-906.

Filbin MT (2003) Myelin-associated inhibitors of axonal regeneration in the adult mammalian CNS. Nat Rev Neurosci 4:703-713.

Guirland C, Buck KB, Gibney JA, DiCicco-Bloom E, Zheng JQ (2003) Direct cAMP signaling through G-protein-coupled receptors mediates growth cone attraction induced by pituitary adenylate cyclase-activating polypeptide. J Neurosci 23:2274-2283.

Han J, Han L, Tiwari P, Wen Z, Zheng JQ (2007) Spatial targeting of type II protein kinase A to filopodia mediates the regulation of growth cone guidance by cAMP. J Cell Biol 176:101-111.

Holz GG, Kang G, Harbeck M, Roe MW, Chepurny OG (2006) Cell physiology of cAMP sensor Epac. J Physiol 577:5-15.

Hong K, Nishiyama M, Henley J, Tessier-Lavigne M, Poo MM (2000) Calcium signalling in the guidance of nerve growth by netrin-1. Nature 403:93-98.

Huber AB, Kolodkin AL, Ginty DD, Cloutier JF (2003) Signaling at the growth cone: ligand-receptor complexes and the control of axon growth and guidance. Annu Rev Neurosci 26:509-563.

Johnson PW, Abramow-Newerly W, Seilheimer B, Sadoul R, Tropak MB, Arquint M, Dunn RJ, Schachner M, Roder JC (1989) Recombinant 
myelin-associated glycoprotein confers neural adhesion and neurite outgrowth function. Neuron 3:377-385.

Kang G, Joseph JW, Chepurny OG, Monaco M, Wheeler MB, Bos JL, Schwede F, Genieser HG, Holz GG (2003) Epac-selective cAMP analog 8-pCPT2 '-O-Me-cAMP as a stimulus for $\mathrm{Ca}^{2+}$-induced $\mathrm{Ca}^{2+}$ release and exocytosis in pancreatic $\beta$-cells. J Biol Chem 278:8279-8285.

Kase H, Iwahashi K, Nakanishi S, Matsuda Y, Yamada K, Takahashi M, Murakata C, Sato A, Kaneko M (1987) K-252 compounds, novel and potent inhibitors of protein kinase $\mathrm{C}$ and cyclic nucleotide-dependent protein kinases. Biochem Biophys Res Commun 142:436-440.

Kawasaki H, Springett GM, Mochizuki N, Toki S, Nakaya M, Matsuda M, Housman DE, Graybiel AM (1998) A family of cAMP-binding proteins that directly activate Rap 1 . Science 282:2275-2279.

Lohof AM, Quillan M, Dan Y, Poo MM (1992) Asymmetric modulation of cAMP activity induces growth cone turning. J Neurosci 12:1253-1261.

Ming GL, Song HJ, Berninger B, Holt CE, Tessier-Lavigne M, Poo MM (1997) cAMP-dependent growth cone guidance by netrin-1. Neuron 19:1225-1235.

Moore SW, Kennedy TE (2006) Protein kinase A regulates the sensitivity if spinal commissural axon turning to netrin- 1 but does not switch between chemoattraction and chemorepulsion. J Neurosci 26:2419-2423.

Mukhopadhyay G, Doherty P, Walsh FS, Crocker PR, Filbin MT (1994) A novel role for myelin-associated glycoprotein as an inhibitor of axonal regeneration. Neuron 13:757-767.

Munck S, Bedner P, Bottaro T, Harz H (2004) Spatiotemporal properties of cytoplasmic cyclic AMP gradients can alter the turning behavior of neuronal growth cones. Eur J Neurosci 19:791-797.

Murray AJ (2008) Pharmacological PKA inhibition: all may not be what it seems. Sci Signal 1:re4.

Murray AJ, Shewan DA (2008) Epac mediates cAMP-dependent axon growth, guidance and regeneration. Mol Cell Neurosci 38:578-588.

Neumann S, Bradke F, Tessier-Lavigne M, Basbaum AI (2002) Regeneration of sensory axons within the injured spinal cord induced by intraganglionic cAMP elevation. Neuron 34:885-893.

Ooashi N, Futatsugi A, Yoshihara F, Mikoshiba K, Kamiguchi H (2005) Cell adhesion molecules regulate $\mathrm{Ca}^{2+}$-mediated steering of growth cones via cyclic AMP and ryanodine receptor type 3. J Cell Biol 170:1159-1167.

Penn RB, Parent JL, Pronin AN, Panettieri RA Jr, Benovic JL (1999) Pharmacological inhibition of protein kinases in intact cells: Antagonism of beta-adrenergic receptor ligand binding by $\mathrm{H} 89$ reveals limitations of usefulness. J Pharmacol Exp Ther 288:428-437.

Pereira L, Metrich M, Fernandez-Velasco M, Lucas A, Leroy J, Perrier R, Morel E, Fischmeister R, Richard S, Benitah JP, Lezoualch F, Gomez AM (2007) Epac modulates $\mathrm{Ca}^{2+}$ spark by $\mathrm{Ca}^{2+} /$ calmodulin kinase signalling pathway in rat cardiac myocytes. J Physiol 583:685-694.

Ponsioen B, Zhao J, Riedl J, Zwartkruis F, van der Krogt G, Zaccolo M, Moolenaar WH, Bos JL, Jalink K (2004) Detecting cAMP-induced Epac activation by fluorescence resonance energy transfer: Epac as a novel cAMP indicator. EMBO Rep 5:1176-1180.
Qiao J, Mei FC, Popov VL, Vergara LA, Cheng X (2002) Cell cycledependent subcellular localization of exchange factor directly activated by cAMP. J Biol Chem 277:26581-26586.

Qiu J, Cai D, Dai H, McAtee M, Hoffman PN, Bregman BS, Filbin MT (2002) Spinal axon regeneration induced by elevation of cyclic AMP. Neuron 34:895-903.

Rehmann H, Schwede F, Døskeland SO, Whittinghoffer A, Bos JL (2003) Ligand-mediated activation of the cAMP-responsive guanine nucleotide exchange factor Epac. J Biol Chem 278:38548-38556.

Seino S, Shibasaki T (2005) PKA-dependent and PKA-independent pathways for cAMP-regulated exocytosis. Physiol Rev 85:1303-1342.

Shewan D, Dwivedy A, Anderson R, Holt CE (2002) Age-related changes underlie switch in netrin-1 responsiveness as growth cones advance along the visual pathway. Nat Neurosci 5:955-962

Song HJ, Poo MM (1999) Signal transduction underlying growth cone responses to diffusible factors. Curr Opin Neurobiol 9:355-363.

Song HJ, Ming GL, Poo MM (1997) cAMP-induced switching in turning direction of nerve growth cones. Nature 388:275-279.

Song H, Ming G, He Z, Lehmann M, McKerracher L, Tessier-Lavigne M, Poo M (1998) Conversion of neuronal growth cone responses from repulsion to attraction by cyclic nucleotides. Science 281:1515-1518.

Terai K, Matsuda M (2006) The amino-terminal B-Raf-specific region mediates calcium dependent homo- and hetero-dimerization of Raf. EMBO J 25:3556-3564.

Tessier-Lavigne M, Goodman CS (1996) The molecular biology of axon guidance. Science 274:1123-1133.

Tojima T, Akiyama H, Itofusa R, Li Y, Katayama H, Miyawaki A, Kamiguchi $\mathrm{H}$ (2007) Attractive axon guidance involves asymmetric membrane transport and exocytosis in the growth cone. Nat Neurosci 10:58-66.

Wen Z, Guirland C, Ming GL, Zheng JQ (2004) A CaMKII/calcineurin switch controls the direction of $\mathrm{Ca}^{2+}$-dependent growth cone guidance. Neuron 43:835-846.

Wong ST, Henley JR, Kanning KC, Huang KH, Bothwell M, Poo MM (2002) A p75NTR and Nogo receptor complex mediates repulsive signaling by myelin-associated glycoprotein. Nat Neurosci 5:1302-1308.

Wouters FS, Verveer PJ, Bastiaens PIH (2001) Imaging biochemistry inside cells. Trends Cell Biol 11:203-211.

Zaccolo M, Pozzan T (2002) Discrete microdomains with high concentration of cAMP in stimulated rat neonatal cardiac myocytes. Science 295:1711-1715.

Zhong J, Li X, McNamee C, Chen AP, Baccarini M, Snider WD (2007) Raf kinase signaling functions in sensory neuron differentiation and axon growth in vivo. Nat Neurosci 10:598-607.

Zhong N, Zucker RS (2005) cAMP acts on exchange protein activated by cAMP/cAMP-regulated guanine nucleotide exchange protein to regulate transmitter release at the crayfish neuromuscular junction. J Neurosci 25:208-214. 